\title{
Article \\ Impact of KMT2A Rearrangement and CSPG4 Expression in Pediatric Acute Myeloid Leukemia
}

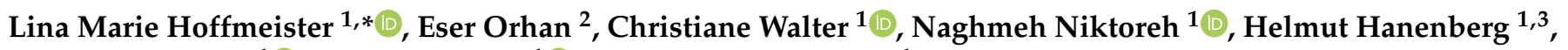 \\ Nils von Neuhoff ${ }^{1} \mathbb{B}$, Dirk Reinhardt ${ }^{1} \mathbb{D}$ and Markus Schneider ${ }^{1, *}$ \\ 1 Department of Pediatric Hematology and Oncology, University Children's Hospital Essen, University of \\ Duisburg-Essen, 45122 Essen, Germany; Christiane.Walter2@uk-essen.de (C.W.); \\ Naghmeh.Niktoreh@uk-essen.de (N.N.); Helmut.Hanenberg@uk-essen.de (H.H.); \\ Nils.vonNeuhoff@uk-essen.de (N.v.N.); dirk.reinhardt@uk-essen.de (D.R.) \\ 2 Centre for Research Acceleration in Pediatrics GmbH, 30175 Hannover, Germany; orhan.eser@aml-bfm.de \\ 3 Department of Otorhinolaryngology and Head/Neck Surgery, Heinrich Heine University, \\ 40225 Düsseldorf, Germany \\ * Correspondence: LinaMarie.Hoffmeister@uk-essen.de (L.M.H.); Markus.Schneider@uk-essen.de (M.S.)
}

check for updates

Citation: Hoffmeister, L.M.; Orhan, E.; Walter, C.; Niktoreh, N.; Hanenberg, H.; von Neuhoff, N.; Reinhardt, D.; Schneider, M. Impact of KMT2A Rearrangement and CSPG4 Expression in Pediatric Acute Myeloid Leukemia. Cancers 2021, 13, 4817. https://doi.org/10.3390/ cancers13194817

Academic Editor: Csongor Kiss

Received: 15 September 2021 Accepted: 22 September 2021 Published: 26 September 2021

Publisher's Note: MDPI stays neutral with regard to jurisdictional claims in published maps and institutional affiliations.

Copyright: (c) 2021 by the authors. Licensee MDPI, Basel, Switzerland. This article is an open access article distributed under the terms and conditions of the Creative Commons Attribution (CC BY) license (https:/ / creativecommons.org/licenses/by/ $4.0 /)$.
Simple Summary: In order to determine the impact of KMT2A rearrangements (KMT2A-r) on the clinical characteristics and treatment outcome of pediatric acute myeloid leukemia (AML) patients, we analyzed a German population-based AML cohort of 967 patients, diagnosed between 2004 and 2019, from which 241 harbored KMT2A-r. KMT2A-r is associated with a higher disease burden and younger age at diagnosis, as well as morphologic subtype of AML M5. The 5-year overall survival rate of patients with KMT2A-r was comparable to those of patients without KMT2A-r. When analyzing AML blasts with KMT2A-r for the presence of additional genetic aberrations using different methods, e.g., classical cytogenetics, next-generation sequencing and multiplex PCR, we found the frequency of KRAS mutations increased, whereas FLT3-ITDs decreased compared to patients without KMT2A-r. Finally, we demonstrated that a correlation between CSPG4 expression and KMT2A-r exists in pediatric AML blasts; however, CSPG4 expression was not specific for blasts with KMT2A-r.

Abstract: $K M T 2 A$ rearrangements $(K M T 2 A-r)$ are among the most common structural aberrations in pediatric acute myeloid leukemia (AML) and are very important for the risk group stratification of patients. Here, we report the outcome of 967 pediatric AML patients with a known KMT2A-r status. The large cohort was characterized by morphology, multicolor flow cytometry, classical cytogenetics and mutation analysis via panel sequencing. In total, the blasts of 241 patients (24.9\%) showed KMT2A-r. KMT2A-r is associated with FAB M5, a high white blood cell count and younger age at diagnosis. When subgroups were combined, KMT2A-r had no impact on event-free survival (EFS) and overall survival (OS); however, various subgroups showed a different prognosis, ranging from a $<50 \%$ OS for KMT2A/AFDN $(n=11)$ to a $100 \%$ chance of survival for patients harboring the rare translocation KMT2A/SEPTIN9 ( $n=3$, follow up of 3.7 to 9.6 years). A positive correlation of $K M T 2 A$-r with $K R A S$ mutations $(p<0.001)$ existed, albeit without any prognostic impact. In addition, FLT3-ITDs were detected less frequently in AML with KMT2A-r $(p<0.001)$. Furthermore, KMT2A-r were mutually exclusive, with mutations in NPM1 $(p=0.002), \operatorname{KIT}(p=0.036)$, WT1 $(p<0.001)$ and CEBPA $(p=0.006)$, and translocations NUP98/NSD1 $(p=0.009), R U N X 1 / R U N X 1 T 1(p=0.003)$ and $C B F B / M Y H 11(p=0.006)$. In the 346 patients tested for CSPG4 expression, a correlation between CSPG4 expression and KMT2A-r was confirmed. However, CSPG4 expression also occurred in patients without $K M T 2 A-r$ and had no significant prognostic impact on EFS and OS.

Keywords: acute myeloid leukemia; childhood acute myeloid leukemia; pediatric; KMT2A; $M L L$; CSPG4; NG2; AML-BFM 


\section{Introduction}

Pediatric acute myeloid leukemia (AML) is a heterogeneous malignancy of the hematopoietic system caused by a variety of different recurrent genetic aberrations that determine the risk group stratification and treatment of the AML patients [1,2]. The overall survival rate for pediatric AML has increased tremendously over the past few decades [3]. Currently, pediatric patients diagnosed with AML have a 5-year overall survival (OS) of $76 \pm 4 \%$, and a 5 -year event-free survival (EFS) is observed in $50 \pm 2 \%$ of the patients [4].

$K M T 2 A$ rearrangements (KMT2A-r) are among the most common recurrent genetic aberrations in pediatric AML, occurring in $24 \%$ of newly diagnosed patients [2]. To date, more than 90 different fusion partner genes of KMT2A have been identified. However, in the setting of pediatric and infant AML, KMT2A/MLLT3 and KMT2A/MLLT10 translocations are among the most frequent KMT2A-r [5]. The most common fusion partner genes of KMT2A are functionally related and interact in a protein complex [6,7]. KMT2A has histone H3lysine-4-methyltransferase activity, and KMT2A fusion proteins recruit the super elongation complex, thereby causing transcriptional elongation [8,9]. As a result, KMT2A-r effects leukemogenesis, for example, by inducing high HOXA gene expression [10,11].

The outcome of patients with KMT2A-rearranged AML is dependent on the fusion partner genes $[12,13]$. Approximately a decade ago, Balgobind et al. studied the outcome of pediatric AML patients with different KMT2A-r in a large, multicentric analyzed cohort [12]. It was shown that patients with $K M T 2 A / A F D N$ translocations had the worst outcomes. Translocation of KMT2A/MLLT10 also showed a poor prognosis, whereas patients with KMT2A/MLLT11 translocations showed an event-free survival rate of $92 \%$ and an overall survival rate of $100 \%$. With an overall survival of $63 \%$, this study showed a favorable prognosis for the most frequent KMT2A fusion partner gene, MLLT3 [12]. The same trends were confirmed in an adult AML cohort [13].

The number of additional aberrations in AML with KMT2A-r is low compared to patients without these aberrations $[10,13]$. However, genes of the RAS pathway, such as KRAS, NRAS and PTPN11, are commonly mutated in AML with KMT2A-r [10,13,14].

CSPG4, previously named human homologue of NG2, is absent on the surface of healthy cells of the hematopoietic system, but is aberrantly expressed on the leukemic blasts of a proportion of pediatric (11-35\%) and adult AML (13-36\%) patients, as well as in ALL [15-19]. The expression level of CSPG4 is heterogeneous between patients, and CSPG4 expression was shown to be strongly associated with KMT2A-r $[15,16,19]$. Although CSPG4 is frequently expressed on the surface of AML cases with KMT2A-r, it is not expressed on the blasts of every AML patient with KMT2A-r. In AML, CSPG4 expression is not dependent on specific fusion partner genes of KMT2A. Both KMT2A-r and CSPG4 expression correlate with the monocytic morphology of blasts $[15,20]$.

To date, most analyzes regarding the impact of KMT2A-r on outcomes in AML have been multicentric, including various treatment protocols [12,21]. Here, we present the clinical, genetic and multicolor flow cytometric data of 967 pediatric AML patients who were enrolled onto one of the German pediatric AML-BFM studies and registries between 2004 and 2019. Since 97\% of all pediatric AML patients who develop leukemia in Germany are enrolled onto one of the AML-BFM studies [3], this cohort is population-based which reflects the German population. Patients were enrolled onto the trials AML04 and AMLS12 and the registries AMLR12 and AMLR17, all of which had comparable treatment protocols. Therefore, we present a large population-based cohort of equally treated pediatric AML patients. We compared the influence of different KMT2A-r on outcomes of treatment and analyzed the presence of additional cytogenetic and molecular genetic aberrations, as well as the co-expression of the surface molecule CSPG4.

\section{Materials and Methods}

\subsection{Study Cohort}

We investigated 967 pediatric AML patients (<18 years) with diagnosis until 2019 (excluding FAB M3 and Down Syndrome) and known KMT2A-r status. All analyzed 
patients were treated in Germany and enrolled onto one of the following AML-BFM trials or registries: AML-BFM 04 trial (ClinicalTrials.gov Identifier: NCT00111345), AML-BFM 2012 trial or registry (EudraCT number: 2013-000018-39) and AML-BFM 2017 registry (DRKS number: DRKS00013030). All trials and registries were approved by the ethical committees and institutional review boards of the university hospitals of Münster, Hannover and Essen in accordance with the declaration of Helsinki. For patients taking part in all trials and registries, written informed consent was obtained before beginning treatment. Treatment protocols of all analyzed trials and registries include similar cytarabine/daunorubicin-based induction chemotherapy. Duration of maintenance treatment and indication of stem cell transplantation in each trial was dependent on the risk group stratification. Standard procedures for diagnostics, especially for morphologic, immune phenotypic and molecular genetic diagnostics, were carried out in the German AML-BFM reference laboratory, except the classical cytogenetic analysis which was performed in the institute for human genetics in Hannover. As 97\% of all German patients diagnosed with pediatric AML are enrolled onto one of the AML-BFM trials, our cohort represents the German population [3].

\subsection{Genetic Data}

Depending on the original trial and time of diagnosis, the KMT2A-r analysis was performed in bone marrow or peripheral blood samples using the following methods: classical karyotyping, fluorescence in situ hybridization or multiplex polymerase chain reaction [22]. In addition, RNA sequencing was carried out in 105 of the 967 patients. RNA sequencing was performed using the TruSight RNA Fusion Panel (Illumina, San Diego, CA, USA) following the manufacturer's recommendations. Sequencing was performed on an Illumina MiSeqDX sequencer in research mode with 76 bp paired-end reads using the MiSeq Reagent Kit v3 (150-cycle). For data analysis, including fusion calling, the RNA Fusion Analysis Module v2.0 of the local run manager of the MiSeqDX system was used. Additional genetic aberrations were identified by fragment length analysis for the detection of FLT3 internal tandem duplications (FLT3-ITD) and mutation screening with the TruSight Myeloid Panel (Illumina, San Diego, CA, USA) (309/967). The sample preparation for mutational screening was prepared according to the manufacturer's instructions. Sequencing was performed on an Illumina MiSeqDX sequencer in research mode with $156 \mathrm{bp}$ paired-end reads using the MiSeq Reagent Kit v2 (300-cycle). For data analysis, SOPHiA $\mathrm{DDM}^{\mathrm{TM}}$ Software was used. Classical karyotyping and fluorescence in situ hybridization were performed in the institute for human genetics in Hannover. The subcohort of patients that was analyzed with mutational screening comprised all patients with diagnosis of AML since 2016 and some randomly selected cases that were retrospectively analyzed (Table S1).

\subsection{Flow Cytometric Data}

Between 2010 and 2018, a subcohort of 346 patients was analyzed for CSPG4 expression via flow cytometry (Supplementary Table S2). The PE conjugated antibody NG2 (clone 7.1) from Beckman Coulter was used for the detection of CSPG4 expression on AML blasts. Leukemic cells were identified as recommended [22]. CSPG4 expression levels were determined and grouped according to the AIEOP-BFM consensus guidelines for antigen expression rating [23].

\subsection{Statistical Analysis}

The Kaplan-Meier method was used to determine the probability of overall (OS) and event-free survival (EFS). As we expect to notice every event of each patient with pediatric AML in Germany, patients without any events were censored at the time of the analysis for both the OS and EFS. OS (or EFS) was determined in years from the diagnosis to death (or first event) or until this analysis. Events were defined as relapse, death, secondary malignancy, non-response to treatment (no complete remission after second induction) as well as early death (<43 days after diagnosis). Early death, as well as non-response, were 
defined as events at diagnosis. Survival curves were compared using the log-rank test. All other statistical calculations were carried out using either an unpaired $t$-test or Pearson's Chi-Square test. Differences with a $p$-value less than 0.05 were considered statistically significant. All statistical analyzes were performed using IBM ${ }^{\circledR}$ SPSS Statistics, version 27. For the generation of diagrams, GraphPad Prism, version 6, was used.

\section{Results}

\subsection{Patient Characteristics}

Our cohort of 967 pediatric AML patients includes 241 patients with KMT2A-r (24.9\%) as shown by classical karyotyping, fluorescence in situ hybridization, multiplex PCR or targeted RNA sequencing and 726 patients without KMT2A-r (75.1\%) (Table 1). Patients with $K M T 2 A-r$ were significantly younger at diagnosis with a median age of 3.3 years, compared to patients who were not carrying KMT2A-r with a median age of 10.0 years $(p<0.001)$. The age distribution of pediatric AML patients (Figure 1a) divides into two main peaks in infancy ( $<12$ months) and adolescence (14-17 years). However, most of the patients with $K M T 2 A-r$ develop leukemia as an infant ( $<12$ months) or at an early childhood age (1-3 years) (Figure $1 b$ ). Patients without KMT2A-r show a more even age distribution $(p<0.001)$. Gender distribution did not differ significantly between the two groups. In patients with KMT2A-r, the white blood cell (WBC) count $(p=0.001)$ and platelet count $(p<0.001)$ at diagnosis were significantly increased compared to patients without KMT2A-r (Table 1). Regarding the morphology of AML blasts, patients with KMT2A-r showed a significant increase in the monocytic morphology-the FAB subtype M5 occurred particularly frequently $(p<0.001)$. Distribution into risk groups was based on the molecular genetics and response to therapy. With respect to previous observations, KMT2A-r strongly influences the stratification into risk groups. The distribution of risk groups therefore significantly differs between patients with and without KMT2A-r $(p<0.001)$ with greatly increased proportions of patients with intermediate and high risk in the group of patients carrying KMT2A-r.

\subsection{KMT2A-r and Subgroups}

A more detailed analysis of the 241 patients with KMT2A-r identified 13 different $K M T 2 A$ fusion partner genes and a group for which the fusion partner gene could not be identified. The latter is, therefore, named KMT2A-r not further specified (Table 2). The most frequent types of KMT2A-r were KMT2A/MLLT3 (43\%, $n=104)$ and KMT2A/MLLT10 (23\%, $n=55)$ fusions, followed by KMT2A/AFDN $(5 \%, n=11)$ and $K M T 2 A / M L L T 1(5 \%, n=11)$. Because of the small number of patients, all other $K M T 2 A$ subgroups were grouped as $K M T 2 A$ other in the analysis of survival curves. KMT2A subgroups and survival data are described in more detail in Table 2.

\subsection{Genetics}

In order to analyze the impact of KMT2A-r on the outcome of pediatric AML patients, the overall survival (OS) and event-free survival (EFS) of the 241 patients with (OS $=72.8 \pm 2.9 \%$; $\mathrm{EFS}=57.5 \pm 3.2 \%)$ and the 726 patients without $K M T 2 A-\mathrm{r}(\mathrm{OS}=75.8 \pm 1.6 \%$; EFS $=58.3 \pm 1.8 \%)$ were compared. This comparison did not show any significant difference between both groups, neither for OS ( $p=0.298$; Figure $2 b$ ) nor EFS ( $p=0.640$; Figure $2 a)$. Separately analyzing the impact of KMT2A-r in patients with standard and intermediate risk or high risk also did not show a significant difference between OS and EFS (Supplementary Figure S1). However, the ratio of early death to other cases of death was significantly higher in AML with KMT2A-r (ratio: 0.38 ) compared to patients without KMT2A-r (ratio: 0.13) $(p=0.002)$. Particularly, the most frequent rearrangements showed high ratios of early death to other cases of death, indicating a severe therapy-independent impact on overall survival: KMT2A/MLLT3 (ratio: 0.44), KMT2A/MLLT10 (ratio: 0.38), KMT2A/AFDN (ratio: 0.50 ) and KMT2A/MLLT1 (ratio: 0.50). Thereby, the WBC count of cases with KMT2A-r and early death was increased accompanied by a higher occurrence of hyperleukocytosis com- 
pared to patients with $K M T 2 A-r$ but without early death. However, the WBC count of cases with early death but without $K M T 2 A-r$ was also increased and hyperleukocytosis occurred more frequently in this group. The infrequent types of KMT2A-r, KMT2A/SEPTIN9 $(n=3)$ and KMT2A/MLLT6 $(n=2)$, did not show a single case of death during a median follow up of 7.0 and 10.6 years, respectively. Additionally, single patients with rare translocations KMT2A/ABI2 ( $n=1$; follow up: 2.6), KMT2A/USP2 ( $n=1$; follow up: 2.1$)$ and KMT2A/KNL1 ( $n=1$; follow up: 6.0$)$ remained event-free during the indicated observation times. Even though the OS of patients with KMT2A-r subgroups did not differ significantly $(p=0.176)$, there was a significant difference between the event-free survival of the different types of KMT2A-r $(p<0.001)$. KMT2A/AFDN translocations showed the lowest probability of 5-year EFS of $9.1 \pm 8.7 \%$, followed by patients with KMT2A/MLLT10 translocations with a 5-year EFS of $43.4 \pm 6.7 \%$. KMT2A/MLLT3 (OS = $77.7 \pm 4.1 \%$; EFS = 66.3 $\pm 4.6 \%$ ); KMT2A/MLLT1 translocations $(\mathrm{OS}=72.7 \pm 13.4 \%$; EFS $=63.6 \pm 14.5 \%)$, together with the group of KMT2A other $(\mathrm{OS}=74.6 \pm 5.7 \%$; EFS $=63.3 \pm 6.2 \%$ ), exhibited the best OS and EFS (Figure 2c, d).

Table 1. Clinical characteristics of pediatric AML patients with and without KMT2A-r at diagnosis.

\begin{tabular}{|c|c|c|c|c|c|}
\hline \multicolumn{2}{|c|}{ Features } & \multirow{2}{*}{$\begin{array}{l}\text { All Patients } \\
967(100 \%)\end{array}$} & \multirow{2}{*}{$\begin{array}{c}\text { Patients with } \\
\text { KMT2A-r } \\
241(25 \%)\end{array}$} & \multirow{2}{*}{$\begin{array}{c}\text { Patients without } \\
\text { KMT2A-r } \\
726(75 \%)\end{array}$} & \multirow{2}{*}{$\begin{array}{c}p \text {-Value } \\
-\end{array}$} \\
\hline Number & & & & & \\
\hline Age & $\begin{array}{l}\text { Median } \\
\text { Range }\end{array}$ & $\begin{array}{c}8.3 \\
0.0-18.0\end{array}$ & $\begin{array}{c}3.3 \\
0.0-17.9\end{array}$ & $\begin{array}{c}10.0 \\
0.0-18.0\end{array}$ & $\begin{array}{c}<0.001^{\mathrm{T}} \\
(* * *)\end{array}$ \\
\hline Gender & $\begin{array}{c}\text { Male } \\
\text { Female }\end{array}$ & $\begin{array}{l}491(100 \%) \\
476(100 \%) \\
\end{array}$ & $\begin{array}{l}134(27 \%) \\
107(22 \%)\end{array}$ & $\begin{array}{l}357(73 \%) \\
369(78 \%)\end{array}$ & $\begin{array}{c}0.084^{\mathrm{C}} \\
\text { (n.s.) }\end{array}$ \\
\hline $\begin{array}{l}\text { WBC count } \\
{\left[\times 10^{3} / \mu \mathrm{L}\right]}\end{array}$ & $\begin{array}{l}\text { Median } \\
\text { Range }\end{array}$ & $\begin{array}{c}16.1 \\
0.0-817.1 \\
\end{array}$ & $\begin{array}{c}19.3 \\
0.2-585.0\end{array}$ & $\begin{array}{c}15.8 \\
0.0-817.1 \\
\end{array}$ & $\begin{array}{c}0.001^{\mathrm{T}} \\
(* *)\end{array}$ \\
\hline $\begin{array}{l}\text { Hemoglobin } \\
{[\mathrm{g} / \mathrm{dL}]}\end{array}$ & $\begin{array}{l}\text { Median } \\
\text { Range }\end{array}$ & $\begin{array}{c}8.3 \\
2.1-18.3 \\
\end{array}$ & $\begin{array}{c}8.3 \\
2.1-17.1\end{array}$ & $\begin{array}{c}8.3 \\
3.1-18.3\end{array}$ & $\begin{array}{c}0.156^{\mathrm{T}} \\
\text { (n.s.) }\end{array}$ \\
\hline $\begin{array}{l}\text { Platelet count } \\
{\left[\times 10^{3} / \mu \mathrm{L}\right]}\end{array}$ & $\begin{array}{l}\text { Median } \\
\text { Range }\end{array}$ & $\begin{array}{c}66.0 \\
2.0-1370.0 \\
\end{array}$ & $\begin{array}{c}89.0 \\
5.0-468.0\end{array}$ & $\begin{array}{c}60.0 \\
2.0-1370.0\end{array}$ & $\begin{array}{c}<0.001^{\mathrm{T}} \\
(* * *)\end{array}$ \\
\hline Risk groups & $\begin{array}{c}\text { Standard risk } \\
\text { Intermediate risk } \\
\text { High risk } \\
\text { No data }\end{array}$ & $\begin{array}{c}244(27 \%) \\
552(61 \%) \\
114(13 \%) \\
57 \\
\end{array}$ & $\begin{array}{c}4(2 \%) \\
185(81 \%) \\
40(17 \%) \\
12\end{array}$ & $\begin{array}{c}240(35 \%) \\
367(54 \%) \\
74(11 \%) \\
45\end{array}$ & $\begin{array}{c}<0.001 \mathrm{C} \\
(* * *) \\
-\end{array}$ \\
\hline $\begin{array}{l}\text { Morphologic subtype } \\
\text { (FAB classification) }\end{array}$ & $\begin{array}{c}\text { M0 } \\
\text { M1 } \\
\text { M2 } \\
\text { M4 } \\
\text { M4 eo } \\
\text { M5 } \\
\text { M6 } \\
\text { M7 } \\
\text { No data }\end{array}$ & $\begin{array}{c}19(2 \%) \\
112(14 \%) \\
175(22 \%) \\
134(17 \%) \\
67(8 \%) \\
198(25 \%) \\
12(1 \%) \\
88(11 \%) \\
162\end{array}$ & $\begin{array}{c}2(1 \%) \\
6(3 \%) \\
4(2 \%) \\
36(18 \%) \\
0(0 \%) \\
147(73 \%) \\
1(0 \%) \\
6(3 \%) \\
39\end{array}$ & $\begin{array}{c}17(\%) \\
106(18 \%) \\
171(28 \%) \\
98(16 \%) \\
67(11 \%) \\
51(8 \%) \\
11(2 \%) \\
82(14 \%) \\
123\end{array}$ & $\begin{array}{c}<0.001^{C} \\
(* * *)\end{array}$ \\
\hline CSPG4 expression & $\begin{array}{c}\text { Strong positive } \\
\text { Weak positive } \\
\text { Negative } \\
\text { No data }\end{array}$ & $\begin{array}{c}31(9 \%) \\
55(16 \%) \\
260(75 \%) \\
621\end{array}$ & $\begin{array}{c}23(27 \%) \\
36(42 \%) \\
26(31 \%) \\
156\end{array}$ & $\begin{array}{c}8(3 \%) \\
19(7 \%) \\
234(90 \%) \\
465\end{array}$ & $\begin{array}{c}<0.001^{C} \\
(* * *) \\
-\end{array}$ \\
\hline
\end{tabular}

${ }^{\mathrm{T}}$ unpaired $t$-test; ${ }^{\mathrm{C}}$ Pearson's chi square test; ${ }^{* *} p<0.01$; ${ }^{* * *} p<0.001 ;$ n.s. not significant. 


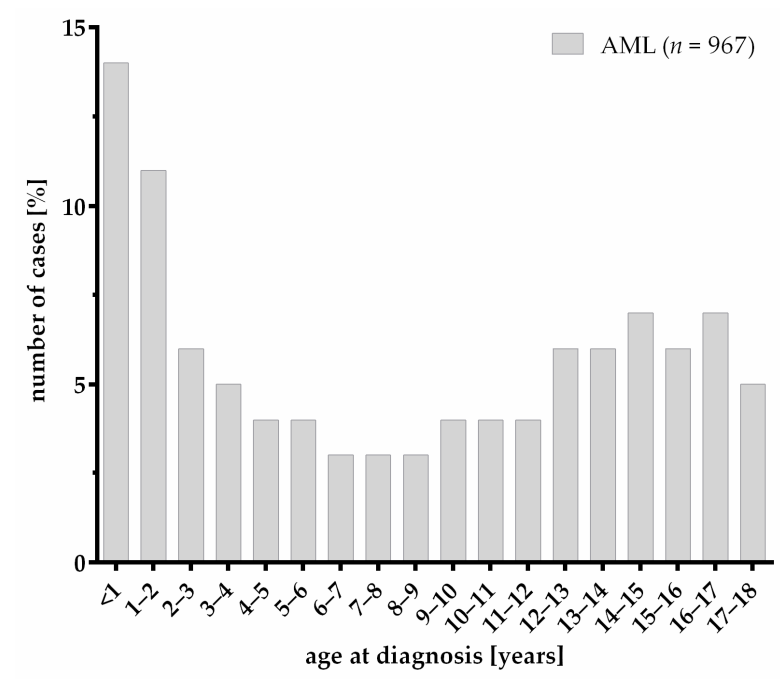

(a)

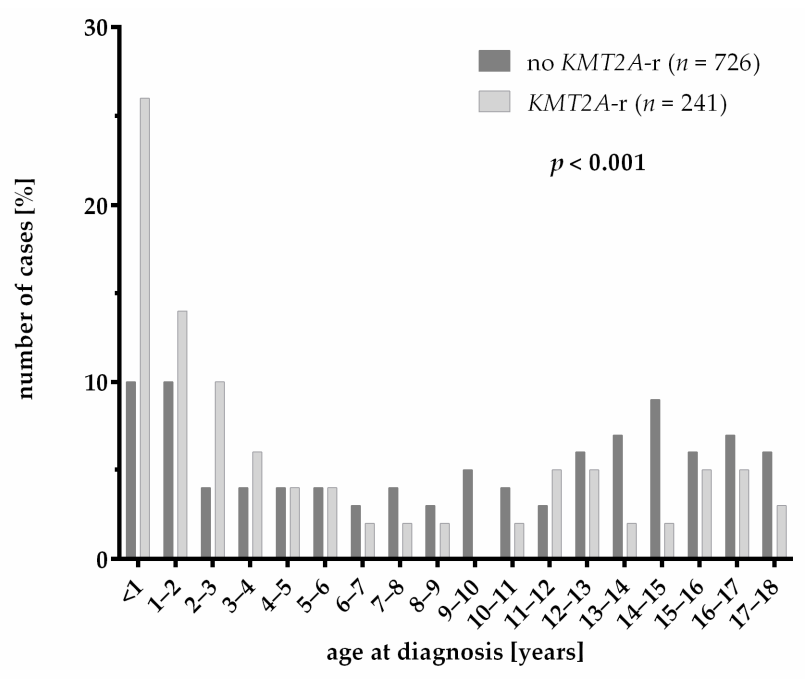

(b)

Figure 1. Age distribution of pediatric AML patients: (a) Age distribution of the total cohort of pediatric AML patients. (b) Comparison of age distribution of pediatric AML patients with and without $K M T 2 A-r(p<0.001)$. Significance was calculated with Pearson's chi square test.

Table 2. Outcome of Pediatric AML Patients with Specific KMT2A-r.

\begin{tabular}{|c|c|c|c|c|c|c|}
\hline Features & Total & Alive & Relapsed & $\begin{array}{l}\text { Dead (Early } \\
\text { Death) }\end{array}$ & $\begin{array}{c}\text { Non- } \\
\text { Response }\end{array}$ & $\begin{array}{l}\text { Secondary } \\
\text { Malignancy }\end{array}$ \\
\hline no $K M T 2 A-r$ & 726 & 547 & 206 & $179(20)$ & 97 & 6 \\
\hline KMT2A/MLLT3 & 104 & 81 & 20 & $23(7)$ & 12 & 1 \\
\hline KMT2A/MLLT10 & 55 & 37 & 16 & $18(5)$ & 12 & 2 \\
\hline KMT2A/AFDN & 11 & 5 & 7 & $6(2)$ & 4 & 0 \\
\hline KMT2A/MLLT1 & 11 & 8 & 3 & $3(1)$ & 1 & 0 \\
\hline KMT2A/MLLT11 & 8 & 7 & 1 & $1(1)$ & 1 & 0 \\
\hline KMT2A/AFF1 & 3 & 2 & 1 & $1(0)$ & 0 & 0 \\
\hline KMT2A/SEPTIN9 & 3 & 3 & 0 & $0(0)$ & 0 & 0 \\
\hline KMT2A/ELL & 3 & 2 & 1 & $1(0)$ & 0 & 0 \\
\hline KMT2A/MLLT6 & 2 & 2 & 0 & $0(0)$ & 0 & 0 \\
\hline KMT2A/PRPF19 & 2 & 1 & 0 & $1(0)$ & 0 & 0 \\
\hline$K M T 2 A / A B I 2$ & 1 & 1 & 0 & $0(0)$ & 0 & 0 \\
\hline KMT2A/KNL1 & 1 & 1 & 1 & $0(0)$ & 0 & 0 \\
\hline KMT2A/USP2 & 1 & 1 & 0 & $0(0)$ & 0 & 0 \\
\hline KMT2A-r not further specified & 36 & 24 & 10 & $12(2)$ & 4 & 0 \\
\hline
\end{tabular}

For the analysis of co-occurrence of additional cytogenetic and molecular genetic aberrations, we analyzed a total of 309 patients that were at least tested with classical cytogenetics and mutational screening with the use of targeted next-generation sequencing. A comparison of the clinical characteristics in this subcohort with the cohort that was not analyzed with panel sequencing revealed a significant bias in age and WBC count, presenting the clinical reality of our studied cohort, which should not have had an effect on the subsequent analyses of the co-occurrence of additional aberrations (Table S1). The additional bias in the distribution of risk groups is probably due to the overall improvements in the detection of variants (fusions + mutations) by next-generation sequencing methods, leading to improved risk group stratification. We found a significant difference in the number of genetic aberrations occurring in patients with and without KMT2A-r ( $p=0.004$; Figure 3). In AML with KMT2A-r, the majority of patients showed only one additional aberration, having a total of two aberrations. In contrast, most patients without KMT2A-r harbored zero to one or more than two aberrations. 


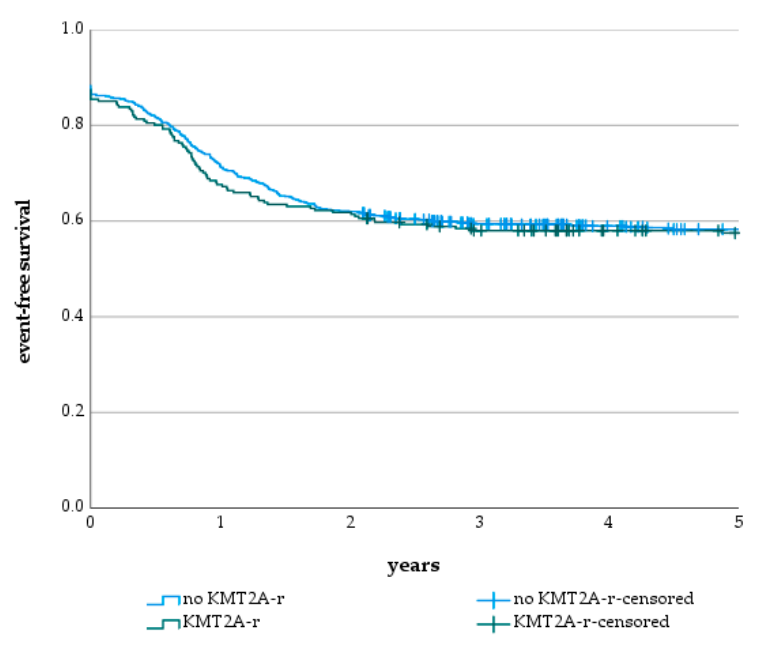

(a)

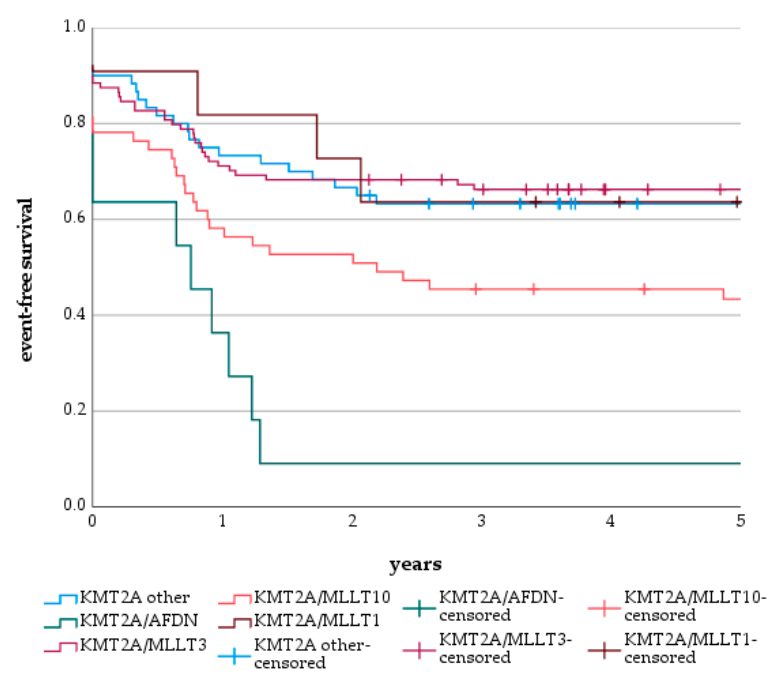

(c)

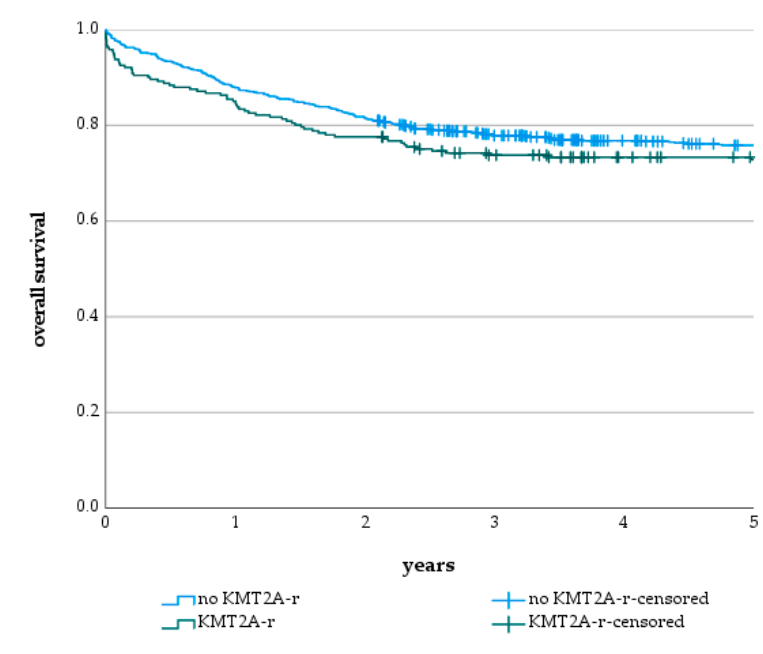

(b)

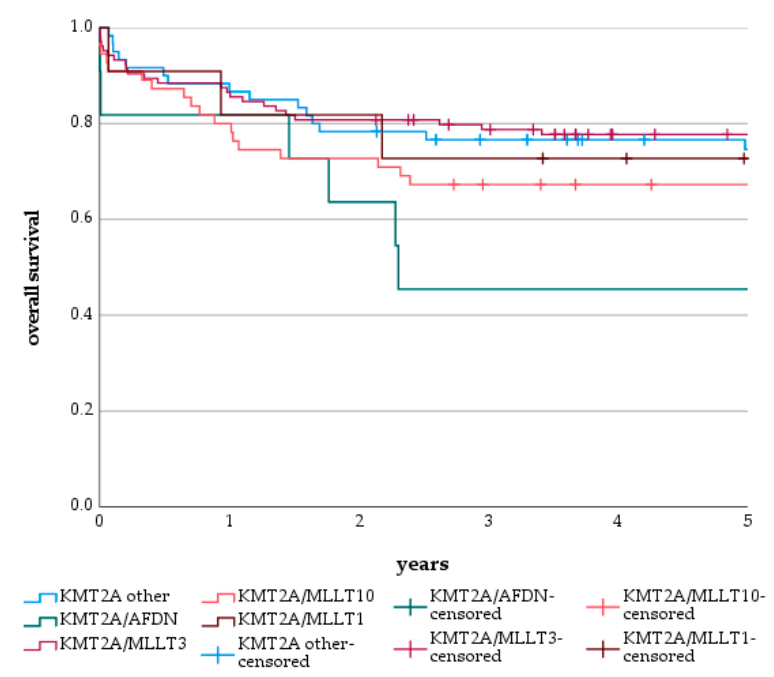

(d)

Figure 2. Survival of pediatric AML patients depending on $K M T 2 A$ rearrangements (KMT2A-r) and subgroups. (a) 5-year event-free survival (EFS) of pediatric AML patients with KMT2A-r (57.5 $\pm 3.2 \%)$ and without KMT2A-r (58.3 $\pm 1.8 \%$ ) $(p=0.640)$; (b) 5-year overall survival (OS) of pediatric AML patients with KMT2A-r (72.8 $\pm 2.9 \%$ ) and without KMT2A-r $(75.8 \pm 1.6 \%)(p=0.298)$; (c) 5-year event-free survival (EFS) of pediatric AML patients with the translocations $K M T 2 A / A F D N$ $(9.1 \pm 8.7 \%)$, KMT2A/MLLT1 (63.6 $\pm 14.5 \%)$, KMT2A/MLLT3 (66.3 $\pm 4.6 \%)$, KMT2A/MLLT10 (43.4 $\pm 6.7 \%)$ or other KMT2A-r $(63.3 \pm 6.2 \%)(p<0.001)$; (d) 5-year overall survival (OS) of pediatric AML patients with the translocations $K M T 2 A / A F D N$ $(45.5 \pm 15.0 \%), K M T 2 A / M L L T 1$ (72.7 $\pm 13.4 \%)$, KMT2A/MLLT3 (77.7 $\pm 4.1 \%)$, KMT2A/MLLT10 (67.3 $\pm 6.3 \%)$ or other KMT2A-r $(74.6 \pm 5.7 \%)(p=0.176)$. Significance was calculated with log-rank test.

We observed a significant positive correlation between $K M T 2 A-r$ and mutations in the KRAS gene $(p<0.001)$. However, no impact of $K R A S$ mutations on the outcome of patients with $K M T 2 A-r$ could be observed (Figure S2). Interestingly, no KRAS mutation was detected in patients with KMT2A/MLLT1 translocation $(n=6)$ (Table S3).

Other genetic aberrations that occurred frequently in AML with KMT2A-r are trisomy 8, NRAS mutations and FLT3-TKD mutations. However, none showed a significant co-occurrence. FLT3-ITDs were found significantly less frequently in AML with KMT2A-r $(p<0.001)$. Additionally, KMT2A-r were mutually exclusive with mutations in NPM1 $(p=0.002), \operatorname{KIT}(p=0.036), W T 1(p<0.001), C E B P A(p=0.006)$ and the translocations NUP98/NSD1 ( $p=0.009)$, RUNX1/RUNX1T1 $(p=0.003)$ and CBFB/MYH11 $(p=0.006)$, con- 
sidering only translocations occurring recurrently in at least 10 patients without KMT2A-r (Table S4).

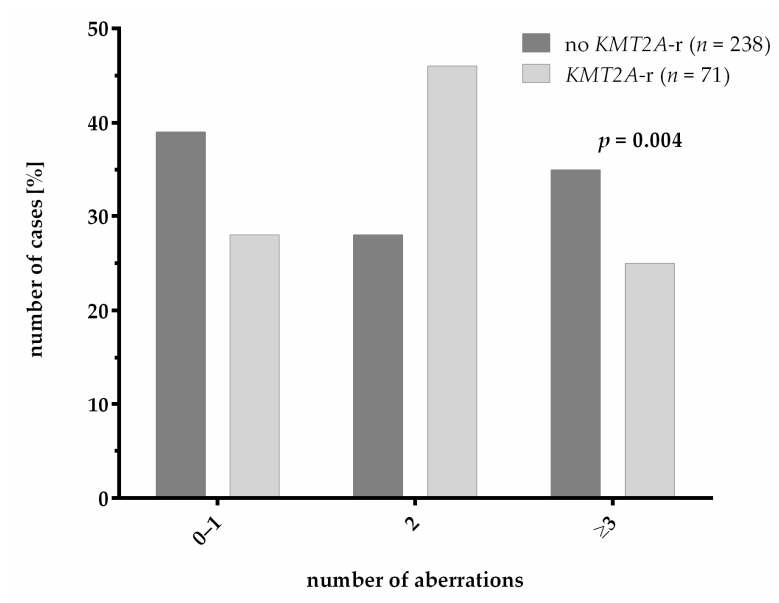

Figure 3. Number of genetic aberrations in pediatric AML patients with and without KMT2A-r $(p=0.004)$. Significance was calculated with Pearson's chi square test.

\subsection{CSPG4 Expression}

Eighty-five patients with and 261 patients without KMT2A-r were analyzed for surface expression of CSPG4 on AML blasts at diagnosis. A comparison of clinical characteristics between the patients analyzed and not analyzed for CSPG4 expression showed a discrepancy in the distribution of risk groups (Table S2). This is probably caused by the continuous improvement of the detection of variants (fusions and mutations), which guides the risk group stratification, during the collection of cases for the CSPG4 expression analysis. The analysis showed that KMT2A-r significantly correlates with CSPG4 expression on the surface of AML blasts $(p<0.001)$ (Table 1$)$. However, not all patients with KMT2A-r showed detectable CSPG4 expression and the expression of CSPG4 was also found on blasts of AML patients without KMT2A-r (Figure 4). Even a strong expression, defined by the detection of CSPG 4 on $>50 \%$ of blasts, was not indicative of KMT2A-r. To elucidate the mechanism causing the aberrant strong ( $>50 \%$ of blasts, $n=8)$ and weak $(10-50 \%$ of blasts, $n=19)$ expression of CSPG4 in cases without KMT2A-r, we analyzed the genetic data of these cases. Interestingly, 3/19 patients with weak CSPG4 expression and 2/8 patients with strong CSPG4 expression showed genetic aberrations in other epigenetic regulators (Table S5). Thus, we were able to identify single cases with weak CSPG4 expression carrying the translocations KAT6A/NCOA2 (1/19) and NUP98/KDM5A (1/19), and one case displaying a $K D M 6 A$ mutation (1/19) without having $K M T 2 A-r$. Additionally, the translocations KAT6A/EP300 (1/8) and KAT6A/CREBBP (1/8) were detected in the cases with strong CSPG4 expression but without $K M T 2 A$-r. Except from aberrations in epigenetic regulators, CBFB/MYH11 translocations (10/19) and WT1 mutations (3/19) were frequent in weak CSPG4-positive cases.

Analysis of the impact of CSPG4 expression levels as a prognostic factor showed neither a significant effect on OS (Figure 5a; $p=0.161$ ) nor on the EFS (Figure 5b; $p=0.204$ ) of the pediatric AML patients. In addition, CSPG4 expression did not have a prognostic significance in AML with KMT2A-r (OS: $p=0.398$, EFS: $p=0.526$ ) (Figure S3). Thus, a strong association for CSPG4 expression on the blasts of pediatric AML with KMT2A-r was identified. However, this CSPG4 expression had no prognostic significance. 


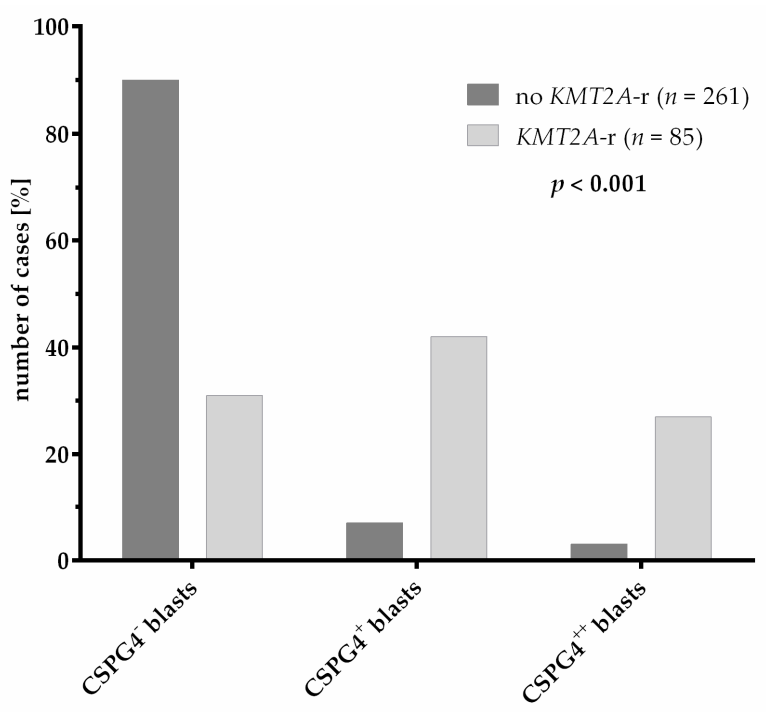

Figure 4. CSPG4 expression levels on blasts of pediatric AML patients with and without KMT2Ar $(p<0.001)$. Significance was calculated with Pearson's chi square test. CSPG4 ${ }^{-}=$no CSPG4 expression ( $<10 \%$ of blasts); CSPG4 ${ }^{+}=$weak CSPG4 expression $\left(10-50 \%\right.$ of blasts); CSPG $4^{++}=$strong CSPG4 expression ( $>50 \%$ of blasts).

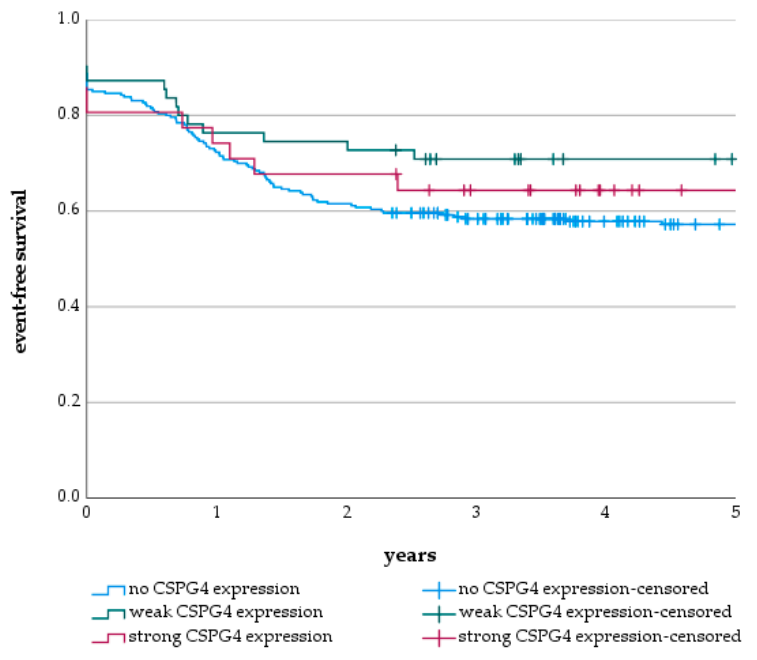

(a)

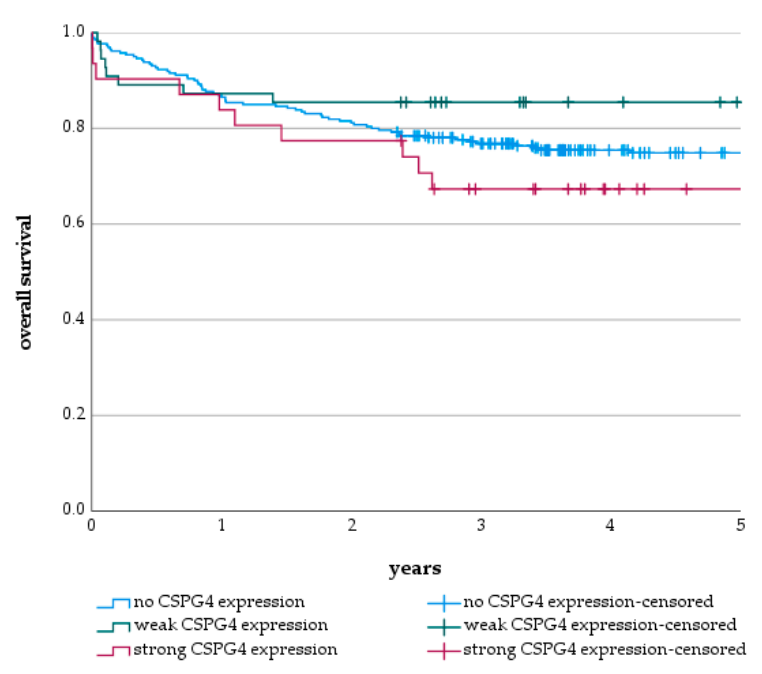

(b)

Figure 5. Survival of pediatric AML patients depending on CSPG4 expression level. (a) 5-year event-free survival (EFS) of pediatric AML patients without CSPG4 expression (57.2 $\pm 3.1 \%$ ), with weak CSPG4 expression level (70.9 $\pm 6.1 \%)$ and with strong CSPG4 expression level $(64.4 \pm 8.6 \%)(p=0.204)$; (b) 5-year overall survival (OS) of pediatric AML patients without CSPG4 expression $(74.9 \pm 2.7 \%)$, with weak CSPG4 expression level (85.5 $\pm 4.8 \%)$ and with strong CSPG4 expression level $(67.3 \pm 8.5 \%)(p=0.161)$. Significance was calculated with log-rank test.

\section{Discussion}

In this work, we present the results of our comparison of a German populationbased cohort of pediatric AML patients with and without KMT2A-r. The parameters analyzed were clinical characteristics, treatment outcome, co-occurrence of additional genetic aberrations and the expression of CSPG4 on the cell surface of AML blasts.

In accordance with previous studies, we showed that pediatric AML patients with KMT2A-r had a younger age and higher WBC count at diagnosis compared to patients without KMT2A-r [20,24]. Regarding the age, we observed that KMT2A-r mostly occur between the ages 0 to 3 years and are mainly responsible for the two-peak age distribu- 
tion of pediatric AML. As previously described, KMT2A-r was strongly associated with monocytic morphology, especially with the FAB subtype M5 [20]. As risk group stratification is strongly based on molecular genetics as well as on therapy response, it was not surprising that the distribution of risk groups correlated with the distribution of KMT2A fusion partner genes [25]. Nevertheless, some differences in risk group stratification and distribution of KMT2A subgroups are due to the availability of newer methods with higher sensitivity (TruSight RNA Fusion Panel) which allowed the retrospective identification of KMT2A translocation partner genes, which would have resulted in a different risk group stratification and, thus, different treatment. Despite the differences in distribution of risks groups, KMT2A-r had no impact on EFS and OS. This underlines the efficiency of the riskadapted treatment approach of the recent protocols, showing remarkable survival rates for patients with high-risk AML mainly resulting from the inclusion of hematopoietic stem cell transplantations (HSCT) as the standard treatment for a high-risk patient accompanied by improved HSCT protocols with less morbidity and mortality over the years $[26,27]$.

In our cohort of 241 pediatric AML patients with KMT2A-r, we identified 13 different translocation partner genes. In line with previous reports, KMT2A/MLLT3 $(43 \%)$ was the most frequent subgroup of KMT2A-r, followed by KMT2A/MLLT10 (23\%), KMT2A/AFDN (5\%) and KMT2A/MLLT1 (5\%) [5]. KMT2A/ELL translocations were less frequently observed in our cohort than in the study of Meyer et al. [5]. In accordance with Balgobind et al., we observed a translocation-partner-gene-dependent difference in the survival outcome of KMT2A-r patients [12]. Compared to the analysis of Balgobind et al. in 2009, we showed improved overall survival rates of all four subgroups of KMT2A-r in our study [12]. We also observed a better event-free survival compared to the analysis of Balgobind et al. Only patients with KMT2A/AFDN translocations also showed a poor event-free survival in our analysis [12]. However, the patient cohort of Balgobind et al. was a multicentric study collecting data from many different countries with different treatment protocols, whereas our study only included patients from Germany receiving similar treatment over many years. Due to the rapid development of a new, more sensitive methodology, especially for molecular genetic diagnostics, risk stratification and, therefore, risk-adapted treatment has been widely improved since the analysis of Balgobind et al. A further major difference between the cohort of Balgobind et al. and our analyzed cohort is the inclusion of HSCT as part of the treatment protocol for high-risk patients. In this regard, Klusmann et al. already showed better survival rates for patients with KMT2A-r allocated to HSCT, which was further confirmed in the study of Rasche et al. [4,26,27]. Here, we were able to discriminate between $K M T 2 A-$ r, which benefits from stratification into a high-risk group (KMT2A/AFDN; KMT2A/MLLT10; KMT2A/AFF1), and those who could be cured with chemotherapy. Therefore, accurate molecular diagnostics at the beginning of the disease is very important to allow risk stratification to be performed correctly; thus, the appropriate treatment protocol can be selected for each patient. Our analysis highlighted that early deaths were more frequent in AML with KMT2A-r compared to non-KMT2Arearranged AML. This was accompanied by a higher WBC count and higher occurrence of hyperleukocytosis. This was also seen in patients with early death but without KMT2A-r. The treatment protocols of this study already include the treatment of patients with high WBC count with hydroxyurea, exchange transfusion or leukapheresis, as the benefit of this treatment was shown by Creutzig et al. in 2015 [28]. Nevertheless, our data suggest that the applied treatment options are insufficient for patients with co-occurrence of high WBC and KMT2A-r and need further adjustments. The underlying mechanism for the higher early death ratio in patients with KMT2A-r compared to patients without KMT2A-r remains elusive and needs additional investigation.

AML with KMT2A-r was previously described to have fewer numbers of genetic aberrations compared to AML with wildtype KMT2A $[10,13]$. In our analysis, AML with KMT2A-r harbored additional mutations in approximately $70 \%$ of cases. However, in accordance with Bill et al., we show that patients with KMT2A-r mostly harbored only one additional aberration whereas patients without $K M T 2 A-r$ more frequently harbored three or 
more aberrations [13]. Our finding that $K R A S$ was frequently mutated in $K M T 2 A-r$ pediatric AML is in line with other studies [10,13,14,29]. Matsuo et al. observed a significant adverse impact of KRAS mutations on the survival of patients with KMT2A-r [29]. However, we did not observe a difference in the outcome of KMT2A-r patients with or without $K R A S$ mutation in our study. Interestingly, previous studies observed a correlation of the occurrence of KMT2A/AFDN translocations with KRAS mutations and higher numbers of additional aberrations. Both were described as adverse prognostic factors, possibly resulting in the severe prognosis that could be observed in our study and in previous studies [29,30]. Unfortunately, in our study, the case number carrying these aberrations was too low $(n=3)$ for an analysis addressing this question (Table S3). NRAS and FLT3-TKD mutations frequently occurred in AML with KMT2A-r, but without statistical significance. This observation was in line with previous studies that identified genes of the RAS-pathway as frequently mutated in AML with KMT2A-r [10,13,14]. In our cohort, FLT3-ITDs occurred less frequently in AML with KMT2A-r. This was in accordance with the observations of Bill et al. [13]. Although trisomy 8 occurred frequently in AML with KMT2A-r in our cohort, we were not able to detect a significant correlation of KMT2A-r and trisomy 8 and the associated significant favorable impact on the survival of these patients due to reduced relapse rates as described by Coenen et al. [30]. In line with previous observations, we found KMT2A-r to be mutually exclusive with RUNX1/RUNX1T1, NUP98/NSD1 and CBFB/MYH11 translocations as well as with NPM1, KIT, WT1 and CEBPA mutations [10]. In contrast, in adult AML with KMT2A-r, Bill et al. described infrequent mutations of NPM1 and CEBPA, underlining the differences between pediatric and adult AML [13].

Surface molecule CSPG4 is not expressed on healthy hematopoietic cells [15]. As the association of KMT2A-r with CSPG4 expression is widely known, its expression is used in diagnostics as the first indicator of KMT2A-r. In the past, CSPG4 was thought to be exclusively expressed on blasts with KMT2A-r [15,16]. Here, we show that the outcome of patients with KMT2A-r is independent from the CSPG4 expression level and that the CSPG4 expression is not exclusive for cases with KMT2A-r independent from the CSPG4 expression level. Thus, CSPG4 cannot be used as a surrogate marker for KMT2A-r in diagnostics. We also observed CSPG4 expression on blasts of patients with point mutations or translocations of other epigenetic regulators such as lysine acetyltransferases or lysine demethylases. The exact mechanism behind the upregulation of CSPG4 expression in AML with KMT2A-r is yet to be determined; nevertheless, some of the aberrations in epigenetic regulators that were found in CSPG4-expressing blasts without KMT2A-r are described to show similar HOXA gene expression profiles to KMT2A-r [31,32]. As wildtype KMT2A and KAT6A interact to activate HOXA9, a similar regulation of CSPG4 expression might be possible [33]. Hence, the question arises, can similar pathways for the regulation of CSPG4 expression be activated? Surprisingly, weak CSPG4 expression with missing KMT2A-r strongly correlated with good prognostic translocation of $C B F B / M Y H 11$. In addition, the blasts of cases with $C B F B / M Y H 11$ showed significantly higher expression levels of $H O X B$ cluster genes and MEIS1 compared to blasts of cases with other favorable aberrations, such as RUNX1/RUNX1T1 [34]. As MEIS1 upregulation seems to be important in AML with KMT2A-r as well as CBFB/MYH11, it is of interest to investigate the downstream regulation of these translocations and the link to the upregulation of CSPG4 further $[34,35]$.

\section{Conclusions}

In summary, in this German population-based study, we have examined the impact of KMT2A-r on the clinical characteristics and outcome of pediatric AML patients, as well as the correlation of KMT2A-r with additional genetic aberrations and the expression of CSPG4 on the cell surface.

Although KMT2A-r associated with the FAB subtype M5, high white blood cell count and younger age at diagnosis, the KMT2A-r status did not influence the OS and EFS. However, a detailed analysis of the KMT2A-r subgroups revealed clear differences in the prognosis of the disease, and also highlighted the importance of refined risk stratification 
groups according to genetic alterations and the successful implementation of HSCT as standard of care for high risk patients. This underlines the importance of accurate molecular diagnostics to successfully identify the different $K M T 2 A$-r subgroups to be able to apply the correct treatment protocol for each patient. In addition, the co-occurrence of KMT2A-r with additional cytogenetic and molecular genetic aberrations further highlighted the heterogeneity of AML with KMT2A-r. Future analysis should address this heterogeneity and its impact on the prognosis of pediatric AML in bigger cohorts, elucidating the underlying mechanisms causing these differences. In this regard, the aberrant expression of CSPG4 and its connection with $K M T 2 A-r$, other epigenetic regulators and $C B F B / M Y H 11$ translocation need further investigation.

Supplementary Materials: The following are available online at https://www.mdpi.com/article/ 10.3390/cancers13194817/s1, Figure S1: Survival of pediatric AML patients depending on KMT2A rearrangements (KMT2A-r) and risk group; Figure S2: Survival of pediatric AML patients with KMT2A-r and with or without KRAS mutation; Figure S3: Survival of pediatric AML patients with KMT2A-r depending on CSPG4 expression level; Table S1: Clinical characteristics of pediatric AML patients with and without mutational screening via next-generation sequencing using the Illumina TruSight Myeloid Panel (TSM); Table S2: Clinical characteristics of pediatric AML patients with and without analysis for CSPG4 expression via multicolor flow cytometry; Table S3: KRAS mutations and correlation with specific KMT2A-r; Table S4: Genetic aberrations and dependency on KMT2A-r status; Table S5: Samples of patients with weak or strong CSPG4 expression and associated gene fusions and mutations.

Author Contributions: Conceptualization, L.M.H., D.R. and M.S.; methodology, L.M.H., E.O., C.W., N.N., H.H., N.v.N., D.R., M.S.; formal analysis, L.M.H., E.O.; investigation, L.M.H., E.O., C.W., N.N., M.S.; resources, N.v.N., D.R.; data curation, L.M.H., E.O., C.W., N.N., M.S.; writing—original draft preparation, L.M.H., M.S.; writing—review and editing, L.M.H., E.O., C.W., N.N., H.H., N.v.N., D.R., M.S.; visualization, L.M.H., E.O.; supervision, H.H., N.v.N., D.R., M.S.; project administration, L.M.H., D.R., M.S.; funding acquisition, N.v.N., D.R. All authors have read and agreed to the published version of the manuscript.

Funding: This research received no external funding.

Institutional Review Board Statement: The study was conducted according to the guidelines of the Declaration of Helsinki, and the retrospective analysis using the data of the patients treated in AMLBFM 2004 and 2012 studies and registries 2012 and 2017 was performed under the ethical approval of the corresponding Institutional Review Boards of the Ethics Committee of Medical Association Westphalen-Lippe and Medical Faculty of WWU Münster (AML-BFM study 2004: protocol code 3VCreutzig1, 5 June 2003), the Ethics Committee of Hannover Medical School (AML-BFM registry 2012: protocol code 1381-2012, 13 March 2012; AML-BFM study 2012: 6741M, 20 November 2014) and the Ethics Committee of the Medical Faculty of University Duisburg-Essen (AML-BFM registry 2017: protocol code 17-7462-BO, 8 December 2017).

Informed Consent Statement: Informed consent was obtained from all subjects involved in the study.

Data Availability Statement: A detailed data sharing statement is provided in the Supplementary Materials.

Acknowledgments: We acknowledge the support by the Essener Elterninitiative zur Unterstützung krebskranker Kinder e.V., the Open Access Publication Fund of the University of Duisburg-Essen and the German Cancer Aid.

Conflicts of Interest: The authors declare no conflict of interest. 


\section{References}

1. Zwaan, C.M.; Kolb, E.A.; Reinhardt, D.; Abrahamsson, J.; Adachi, S.; Aplenc, R.; De Bont, E.S.J.M.; de Moerloose, B.; Dworzak, M.; Gibson, B.E.S.; et al. Collaborative Efforts Driving Progress in Pediatric Acute Myeloid Leukemia. J. Clin. Oncol. 2015, 33, 2949-2962. [CrossRef] [PubMed]

2. Balgobind, B.V.; Hollink, I.H.I.M.; Arentsen-Peters, S.T.C.J.M.; Zimmermann, M.; Harbott, J.; Beverloo, H.B.; von Bergh, A.R.M.; Cloos, J.; Kaspers, G.J.L.; de Haas, V.; et al. Integrative analysis of type-I and type-II aberrations underscores the genetic heterogeneity of pediatric acute myeloid leukemia. Haematologica 2011, 96, 1478-1487. [CrossRef] [PubMed]

3. Erdmann, F.; Kaatsch, P.; Grabow, D.; Spix, C. German Childhood Cancer Registry-Annual Report 2019 (1980-2018). Available online: https:/ / www.kinderkrebsregister.de/dkkr/ergebnisse/jahresberichte/jahresbericht-2019.html (accessed on 12 July 2021).

4. Rasche, M.; Zimmermann, M.; Borschel, L.; Bourquin, J.-P.; Dworzak, M.; Klingebiel, T.; Lehrnbecher, T.; Creutzig, U.; Klusmann, J.-H.; Reinhardt, D. Successes and challenges in the treatment of pediatric acute myeloid leukemia: A retrospective analysis of the AML-BFM trials from 1987 to 2012. Leukemia 2018, 32, 2167-2177. [CrossRef]

5. Meyer, C.; Burmeister, T.; Gröger, D.; Tsaur, G.; Fechina, L.; Renneville, A.; Sutton, R.; Venn, N.C.; Emerenciano, M.; Pombo-deOliveira, M.S.; et al. The MLL recombinome of acute leukemias in 2017. Leukemia 2018, 32, 273-284. [CrossRef]

6. Krivtsov, A.V.; Armstrong, S.A. MLL translocations, histone modifications and leukaemia stem-cell development. Nat. Rev. Cancer 2007, 7, 823-833. [CrossRef]

7. Erfurth, F.; Hemenway, C.S.; de Erkenez, A.C.; Domer, P.H. MLL fusion partners AF4 and AF9 interact at subnuclear foci. Leukemia 2004, 18, 92-102. [CrossRef]

8. Milne, T.A.; Briggs, S.D.; Brock, H.W.; Martin, M.E.; Gibbs, D.; Allis, C.D.; Hess, J.L. MLL Targets SET Domain Methyltransferase Activity to Hox Gene Promoters. Mol. Cell 2002, 10, 1107-1117. [CrossRef]

9. Krivtsov, A.V.; Hoshii, T.; Armstrong, S.A. Mixed-Lineage Leukemia Fusions and Chromatin in Leukemia. Cold Spring Harb. Perspect. Med. 2017, 7, a026658. [CrossRef]

10. Bolouri, H.; Farrar, J.E.; Triche, T.; Ries, R.E.; Lim, E.L.; Alonzo, T.A.; Ma, Y.; Moore, R.; Mungall, A.J.; Marra, M.A.; et al. The molecular landscape of pediatric acute myeloid leukemia reveals recurrent structural alterations and age-specific mutational interactions. Nat. Med. 2018, 24, 103-112. [CrossRef]

11. Armstrong, S.A.; Staunton, J.E.; Silverman, L.B.; Pieters, R.; den Boer, M.L.; Minden, M.D.; Sallan, S.E.; Lander, E.S.; Golub, T.R.; Korsmeyer, S.J. MLL translocations specify a distinct gene expression profile that distinguishes a unique leukemia. Nat. Genet. 2002, 30, 41-47. [CrossRef] [PubMed]

12. Balgobind, B.V.; Raimondi, S.C.; Harbott, J.; Zimmermann, M.; Alonzo, T.A.; Auvrignon, A.; Beverloo, H.B.; Chang, M.; Creutzig, U.; Dworzak, M.N.; et al. Novel prognostic subgroups in childhood 11q23/MLL-rearranged acute myeloid leukemia: Results of an international retrospective study. Blood 2009, 114, 2489-2496. [CrossRef] [PubMed]

13. Bill, M.; Mrózek, K.; Kohlschmidt, J.; Eisfeld, A.-K.; Walker, C.J.; Nicolet, D.; Papaioannou, D.; Blachly, J.S.; Orwick, S.; Carroll, A.J.; et al. Mutational landscape and clinical outcome of patients with de novo acute myeloid leukemia and rearrangements involving 11q23/KMT2A. Proc. Natl. Acad. Sci. USA 2020, 117, 26340-26346. [CrossRef] [PubMed]

14. Grossmann, V.; Schnittger, S.; Poetzinger, F.; Kohlmann, A.; Stiel, A.; Eder, C.; Fasan, A.; Kern, W.; Haferlach, T.; Haferlach, C. High incidence of RAS signalling pathway mutations in MLL-rearranged acute myeloid leukemia. Leukemia 2013, 27, 1933-1936. [CrossRef]

15. Smith, F.O.; Rauch, C.; Williams, D.E.; March, C.J.; Arthur, D.; Hilden, J.; Lampkin, B.C.; Buckley, J.D.; Buckley, C.V.; Woods, W.G.; et al. The human homologue of rat NG2, a chondroitin sulfate proteoglycan, is not expressed on the cell surface of normal hematopoietic cells but is expressed by acute myeloid leukemia blasts from poor-prognosis patients with abnormalities of chromosome band 11q23. Blood 1996, 87, 1123-1133. [PubMed]

16. Hilden, J.M.; Smith, F.O.; Frestedt, J.L.; McGlennen, R.; Howells, W.B.; Sorensen, P.H.; Arthur, D.C.; Woods, W.G.; Buckley, J.; Bernstein, I.D.; et al. MLL gene rearrangement, cytogenetic 11q23 abnormalities, and expression of the NG2 molecule in infant acute myeloid leukemia. Blood 1997, 89, 3801-3805. [CrossRef]

17. Behm, F.G.; Smith, F.O.; Raimondi, S.C.; Pui, C.H.; Bernstein, I.D. Human homologue of the rat chondroitin sulfate proteoglycan, NG2, detected by monoclonal antibody 7.1, identifies childhood acute lymphoblastic leukemias with $\mathrm{t}(4 ; 11)(\mathrm{q} 21 ; \mathrm{q} 23)$ or $\mathrm{t}(11 ; 19)(\mathrm{q} 23 ; \mathrm{p} 13)$ and MLL gene rearrangements. Blood 1996, 87, 1134-1139. [CrossRef] [PubMed]

18. Wuchter, C.; Harbott, J.; Schoch, C.; Schnittger, S.; Borkhardt, A.; Karawajew, L.; Ratei, R.; Ruppert, V.; Haferlach, T.; Creutzig, U.; et al. Detection of acute leukemia cells with mixed lineage leukemia (MLL) gene rearrangements by flow cytometry using monoclonal antibody 7.1. Leukemia 2000, 14, 1232-1238. [CrossRef]

19. Mauvieux, L.; Delabesse, E.; Bourquelot, P.; Radford-Weiss, I.; Bennaceur, A.; Flandrin, G.; Valensi, F.; MacIntyre, E.A. NG2 expression in MLL rearranged acute myeloid leukaemia is restricted to monoblastic cases. Br. J. Haematol. 1999, 107, 674-676. [CrossRef]

20. Cimino, G.; Rapanotti, M.C.; Elia, L.; Biondi, A.; Fizzotti, M.; Testi, A.M.; Tosti, S.; Croce, C.M.; Canaani, E.; Mandelli, F. ALL-1 gene rearrangements in acute myeloid leukemia: Association with M4-M5 French-American-British classification subtypes and young age. Cancer Res. 1995, 55, 1625-1628.

21. Emerenciano, M.; Meyer, C.; Mansur, M.B.; Marschalek, R.; Pombo-de-Oliveira, M.S. The distribution of MLL breakpoints correlates with outcome in infant acute leukaemia. Br. J. Haematol. 2013, 161, 224-236. [CrossRef] 
22. Creutzig, U.; van den Heuvel-Eibrink, M.M.; Gibson, B.; Dworzak, M.N.; Adachi, S.; de Bont, E.; Harbott, J.; Hasle, H.; Johnston, D.; Kinoshita, A.; et al. Diagnosis and management of acute myeloid leukemia in children and adolescents: Recommendations from an international expert panel. Blood 2012, 120, 3187-3205. [CrossRef] [PubMed]

23. Dworzak, M.N.; Buldini, B.; Gaipa, G.; Ratei, R.; Hrusak, O.; Luria, D.; Rosenthal, E.; Bourquin, J.-P.; Sartor, M.; Schumich, A.; et al. AIEOP-BFM consensus guidelines 2016 for flow cytometric immunophenotyping of Pediatric acute lymphoblastic leukemia. Cytometry B Clin. Cytom. 2018, 94, 82-93. [CrossRef] [PubMed]

24. Hara, Y.; Shiba, N.; Yamato, G.; Ohki, K.; Tabuchi, K.; Sotomatsu, M.; Tomizawa, D.; Kinoshita, A.; Arakawa, H.; Saito, A.M.; et al. Patients aged less than 3 years with acute myeloid leukaemia characterize a molecularly and clinically distinct subgroup. Br. J. Haematol. 2020, 188, 528-539. [CrossRef] [PubMed]

25. Rasche, M.; Zimmermann, M.; Steidel, E.; Alonzo, T.; Aplenc, R.; Bourquin, J.-P.; Boztug, H.; Cooper, T.; Gamis, A.S.; Gerbing, R.B.; et al. Survival Following Relapse in Children with Acute Myeloid Leukemia: A Report from AML-BFM and COG. Cancers 2021, 13, 2336. [CrossRef]

26. Rasche, M.; Steidel, E.; Kondryn, D.; von Neuhoff, N.; Sramkova, L.; Creutzig, U.; Dworzak, M.; Reinhardt, D. Impact of a Risk-Adapted Treatment Approach in Pediatric AML: A Report of the AML-BFM Registry 2012. Blood 2019, 134, 293. [CrossRef]

27. Klusmann, J.-H.; Reinhardt, D.; Zimmermann, M.; Kremens, B.; Vormoor, J.; Dworzak, M.; Creutzig, U.; Klingebiel, T. The role of matched sibling donor allogeneic stem cell transplantation in pediatric high-risk acute myeloid leukemia: Results from the AML-BFM 98 study. Haematologica 2012, 97, 21-29. [CrossRef]

28. Creutzig, U.; Rössig, C.; Dworzak, M.; Stary, J.; von Stackelberg, A.; Wössmann, W.; Zimmermann, M.; Reinhardt, D. Exchange Transfusion and Leukapheresis in Pediatric Patients with AML With High Risk of Early Death by Bleeding and Leukostasis. Pediatr. Blood Cancer 2016, 63, 640-645. [CrossRef]

29. Matsuo, H.; Yoshida, K.; Nakatani, K.; Harata, Y.; Higashitani, M.; Ito, Y.; Kamikubo, Y.; Shiozawa, Y.; Shiraishi, Y.; Chiba, K.; et al. Fusion partner-specific mutation profiles and KRAS mutations as adverse prognostic factors in MLL-rearranged AML. Blood Adv. 2020, 4, 4623-4631. [CrossRef]

30. Coenen, E.A.; Raimondi, S.C.; Harbott, J.; Zimmermann, M.; Alonzo, T.A.; Auvrignon, A.; Beverloo, H.B.; Chang, M.; Creutzig, U.; Dworzak, M.N.; et al. Prognostic significance of additional cytogenetic aberrations in 733 de novo pediatric 11q23/MLLrearranged AML patients: Results of an international study. Blood 2011, 117, 7102-7111. [CrossRef]

31. Camós, M.; Esteve, J.; Jares, P.; Colomer, D.; Rozman, M.; Villamor, N.; Costa, D.; Carrió, A.; Nomdedéu, J.; Montserrat, E.; et al. Gene expression profiling of acute myeloid leukemia with translocation $\mathrm{t}(8 ; 16)(\mathrm{p} 11 ; \mathrm{p} 13)$ and MYST3-CREBBP rearrangement reveals a distinctive signature with a specific pattern of HOX gene expression. Cancer Res. 2006, 66, 6947-6954. [CrossRef]

32. de Rooij, J.D.E.; Hollink, I.H.I.M.; Arentsen-Peters, S.T.C.J.M.; van Galen, J.F.; Berna Beverloo, H.; Baruchel, A.; Trka, J.; Reinhardt, D.; Sonneveld, E.; Zimmermann, M.; et al. NUP98/JARID1A is a novel recurrent abnormality in pediatric acute megakaryoblastic leukemia with a distinct HOX gene expression pattern. Leukemia 2013, 27, 2280-2288. [CrossRef] [PubMed]

33. Paggetti, J.; Largeot, A.; Aucagne, R.; Jacquel, A.; Lagrange, B.; Yang, X.-J.; Solary, E.; Bastie, J.-N.; Delva, L. Crosstalk between leukemia-associated proteins MOZ and MLL regulates HOX gene expression in human cord blood CD34+ cells. Oncogene 2010, 29, 5019-5031. [CrossRef] [PubMed]

34. Andreeff, M.; Ruvolo, V.; Gadgil, S.; Zeng, C.; Coombes, K.; Chen, W.; Kornblau, S.; Barón, A.E.; Drabkin, H.A. HOX expression patterns identify a common signature for favorable AML. Leukemia 2008, 22, 2041-2047. [CrossRef] [PubMed]

35. Wong, P.; Iwasaki, M.; Somervaille, T.C.P.; So, C.W.E.; So, C.W.E.; Cleary, M.L. Meis1 is an essential and rate-limiting regulator of MLL leukemia stem cell potential. Genes Dev. 2007, 21, 2762-2774. [CrossRef] [PubMed] 